\title{
La estructura conceptual del registro bibliográfico: una revisión
}

\author{
José Antonio Frías \\ Universidad de Salamanca \\ Facultad de Traducción y Documentación
}

\subsection{Resumen}

Se parte del supuesto de la necesidad de que la estructura conceptual de los registros bibliográficos esté basada en el conocimiento de las necesidades del usuario. En este sentido, la aplicación del modelo entidad-relación al catálogo de la biblioteca permite considerar a los registros bibliográficos y de autoridad como entidades, a los elementos de datos de los registros como atributos y a los vínculos entre los registros y entre éstos y los elementos como relaciones. Se exponen las definiciones de relaciones bibliográficas del formato UNIMARC, el análisis de las relaciones jerárquicas realizado por Goossens y Mazur-Rzesos, la tipología de los documentos bibliográficos establecida por McCallum y se presentan los siete tipos de relaciones desarrolladas por Tillett: de equivalencia, derivativas, descriptivas, todo-parte, de acompañamiento, secuenciales y de características compartidas. Finalmente, se exponen los resultados de un estudio empírico llevado a cabo para determinar la extensión y las características de las relaciones bibliográficas en el catálogo automatizado de la Library of Congress. (Autor)

Palabras clave: Registros bibliográfico. Estructura conceptual. Modelo entidad-relación. Relaciones bibliográficas. Instrumentos de enlace. Reglas de catalogación. Formato MARC.

\subsection{Abstract}

It is assumed that the conceptual structure of bibliographic records is based on the knowledge of the user needs. In this sense, the application of the entityrelationship model to the library catalogue allows us to consider the authoritaty and bibliographic records as entities, data elements in the bibliographic records as attributes, and the links between records and between records and elements as relationships. We point out the bibliographic relationships of UNIMARC format, the analysis of hierarchical relationships carried out by Goossens and MazurRzesos, the tipology of bibliographic documents established by McCallum; we 
also present the seven types of relationships developed by Tillett: equivalence, derivative, descriptive, whole part (or part whole), accompanying, sequential, and shared characteristic relationships. Then, we give the results of an empirical study carried out to establish the extent and features of bibliographic relationships in the computer-based catalogue of the Library of Congress. (Author)

Keywords: Bibliographic records. Conceptual structure. Entity-relationship model. Bibliographic relationship. Linking devices. Cataloguing rules. MARC format.

\section{Introducción: la estructura conceptual del registro bibliográfico}

Uno de los problemas de algunos estudios de uso del catálogo que intentan determinar el conjunto de elementos de datos necesarios en los registros bibliográficos es, según Holley, que consideran a los habitantes del universo bibliográfico como entidades discretas, más que como integrantes de un sistema galáctico interconectado. En consecuencia, no consideran los elementos de datos cuya principal función es organizar el catálogo, es decir, suministrar estructura (Holley, 1992, p. 65-66).

La estructura conceptual de un catálogo describe lo que debe incluirse en éste, los elementos necesarios para describir los documentos y el orden en que deben ser presentados (por ejemplo, el orden prescrito por la ISBD), así como los vínculos o enlaces que deben crearse entre los documentos incluídos (Tillett, 1988, p. 3).

Nuestra visión actual de la estructura conceptual del catálogo puede ser trazada al menos desde una fecha ya tan lejana como mediados del siglo XIX cuando Panizzi defendió firmemente el catálogo de la biblioteca como el instrumento vital para acceder a sus fondos. Sus reglas reflejan una estructura conceptual basada en los asientos, es decir, en registros únicos y completos para cada documento catalogado, y tres clases de referencias cruzadas para vincular el asiento elegido por el usuario con el asiento del catálogo. La estructura conceptual del catálogo consiste, por tanto, en "registros", compuestos de "elementos de datos" y de "conexiones" entre los registros. Los registros conectados forman "agrupaciones" que comparten un tipo particular de relación. Los registros se elaboran para describir entidades bibliográficas y para controlar las materias y los nombres usados como puntos de acceso, incluyendo nombres personales, corporativos, geográficos y de congresos, así como a los títulos, sobre todo los títulos de obras o partes de obras (Tillett, 1989, p. 150).

Los sistemas convencionales de recuperación de la información bibliográfica han sido diseñados, al menos implícitamente, para incorporar sólo una capacidad modesta para relacionar datos. Las relaciones de los datos están basadas en el 
concepto de relacionar términos y descripciones de los registros de los documentos bibliográficos individuales y no hay una vinculación efectiva entre estos registros y otros que comparten elementos o descripciones comunes. La estructura de la base de datos está diseñada para relacionar sólo documentos bibliográficos. No está diseñada para relacionar, por ejemplo, a los editores. Como ha señalado Koenig (1985, p. 249), "ésta es una asunción implícita de que todos los usuarios van a usar el fichero de una manera similar: para obtener acceso a los registros que describen documentos bibliográficos".

De ahí que Leazer (1992, p. 204) piense que "el desarrollo de un plan conceptual para las bases de datos bibliográficos debe estar basado en el conocimiento de las necesidades de los usuarios". Siguiendo un análisis de las necesidades de los usuarios, puede crearse un esquema conceptual claro para la organización y la estructura de las bases de datos bibliográficos de todo tipo.

Un modelo conceptual popular en la actualidad es el modelo entidad-relación, una estructura que incluye entidades, atributos y relaciones. Este modelo, basado en la teoría de conjuntos y en la teoría de la relación, tiene la ventaja de combinar una técnica diagramática con una aproximación semántica a la descripción de los datos o la información y al diseño de las bases de datos. Las entidades son descritas como "cualquier clase de objetos o acontecimientos del mundo real que comparten similares características o propiedades" (Amat, Pérez Salinas y Ramos Pérez, 1989, p. 66). Estas entidades son caracterizadas por atributos que las describen y son comunes a los miembros de un conjunto de entidades y además pueden ser usados como una prueba de pertenencia. Por ejemplo, en el análisis de una biblioteca, los atributos de los miembros del conjunto de entidades "documento" incluye "ISBN" y "título". Un atributo puede ser compartido por uno o más conjuntos de entidades: "título" es además un atributo de "serie", pero otro será "ISSN" y no "ISBN" (Griffiths, 1989, p. 13). Las relaciones son "cualquier clase de hechos elementales o asociaciones entre entidades" (Amat, Pérez Salinas y Ramos Pérez, 1989, p. 66).

Fidel y Crandall (1988, p. 128) han analizado las AACR2 como esquema de diseño de una base de datos y han ofrecido una representación gráfica del modelo-entidad relación para algunos elementos en un registro bibliográfico, que hemos adaptado en la figura 1. Esta figura es un ejemplo de representación gráfica de unas pocas entidades del mundo bibliográfico con sus relaciones y atributos que puede ser útil para ilustrar la naturaleza del diagrama entidad-relación. En la figura las entidades están representadas por recuadros rectangulares, las relaciones están representadas por recuadros romboidales (por ejemplo, "editado por" es una relación entre las entidades "obra” y "editor") y los atributos están representados por círculos ("nombre", por ejemplo, es un atributo de la entidad 


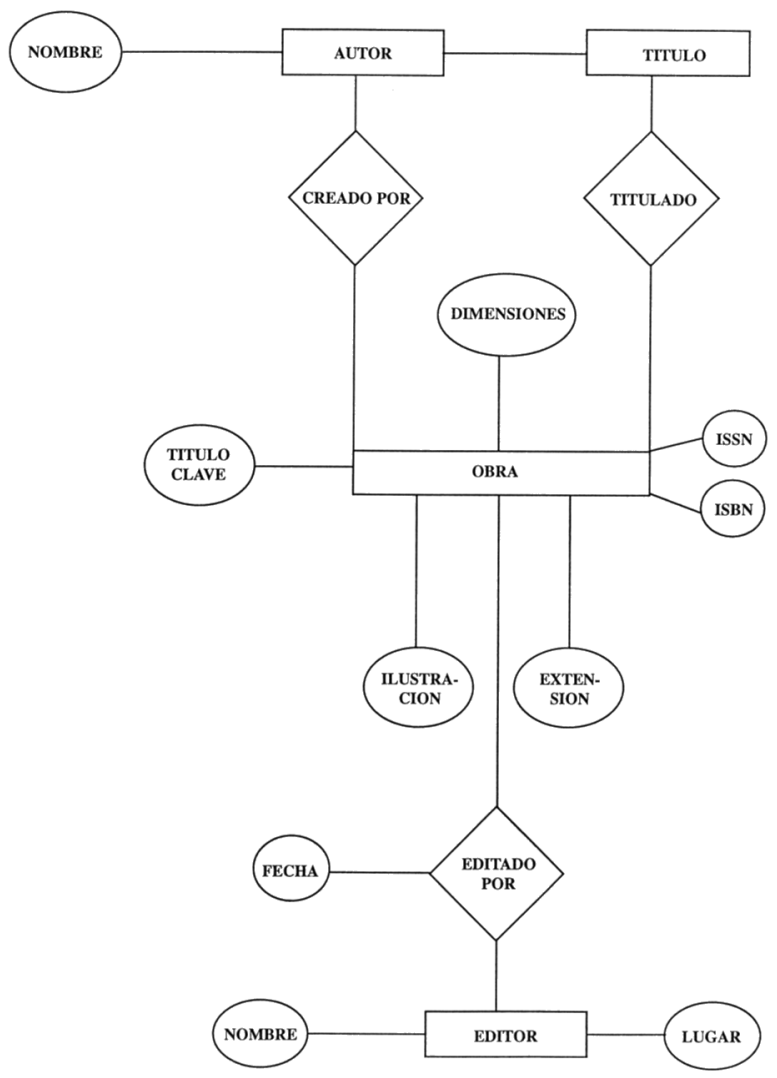

Fig. 1. Una presentación gráfica del modelo entidad-relación para algunos elementos de un registro bibliográfico.

"editor", como también lo es "lugar" mientras "fecha de publicación", sin embargo, es un atributo de la relación "editado por").

Attig (1989, p. 142) ha señalado que existen relaciones de todo tipo que son significativas en la catalogación descriptiva: relaciones bibliográficas (analíticas, traducciones y revisiones), relaciones de autoridad y relaciones de fondos y localizaciones. Todas ellas están implicadas en el establecimiento y el mantenimiento de vínculos entre registros independientes. 
Una posible interpretación de este modelo entidad-relación aplicado al catálogo de la biblioteca es la consideración de los registros bibliográficos y de los registros de autoridad como entidades, de los elementos de los registros como atributos y de los vínculos entre registros y entre éstos y los elementos como relaciones. En un modelo de este tipo, las relaciones pueden ser de cuatro tipos: relaciones bibliográficas, relaciones de nombres, relaciones de materias y relaciones de puntos de acceso. Las reglas de catalogación han cubierto las relaciones bibliográficas y las relaciones de nombres, y las reglas de Cutter cubrían incluso las relaciones entre términos de materia (Tillett, 1988, p. 3).

Un problema no resuelto tradicionalmente en la teoría de la catalogación ha sido la identificación de la obra como unidad de descripción y como soporte de las relaciones bibliográficas en las que se funda "la organicidad y la sistematicidad de la configuración particular de la estructura informativa constituida por el catálogo" (Grimaldi, 1993, p. 134).

Lubetzky (1985, p. 189) escribió al respecto que "el libro... aparece como un producto dicotómico: como un objeto material o medio utilizado para transmitir la obra intelectual de un autor". De este modo, una obra es vista como la entidad intelectual y un documento es visto como el medio físico a través del cual se transmite la obra. La entidad bibliográfica, por tanto, refleja tanto las propiedades físicas del documento como las propiedades intelectuales de la obra, y forma la entidad total que es la base para la descripción bibliográfica (Vellucci, 1990, p. 41).

Una atención renovada hacia la naturaleza y las características de la unidad informativa catalográfica ha venido determinada por la potencialidad de las nuevas tecnologías (y por la lógica del ordenador) de descomponer la rígida estructura monodimensional de los datos catalográficos y de articular una compleja red de relaciones en torno a una unidad informativa de base, con el fin de determinar un formato descriptivo constituido por datos integrables de acuerdo a los atributos bibliográficos que quieran indicarse en repuesta a determinadas exigencias de los usuarios (Grimaldi, 1993, p. 134).

O’Neill y Vizine-Goetz (1989) han propuesto una estructura que es definida jerárquicamente en términos de obras, textos, ediciones, impresiones y libros. Las obras, textos, ediciones e impresiones representan conjuntos de documentos bibliográficos derivados por subdivisiones sucesivas. La definición de entidades de este tipo es fundamental para organizar el universo bibliográfico, ya que la literatura sobre biblioteconomía y bibliografía, incluyendo los propios códigos de catalogación, presenta una confusión y un desacuerdo considerables a este respecto. Sus definiciones se basan en la premisa de que las distinciones bibliográficas deben basarse en una realidad bibliográfica. En su opinión, no son admisi-

Scire. 2 : 2 (jul.-dic. 1996) 53-77 
bles consideraciones extrabibliográficas del tipo de si una traducción que modifica sustancialmente la obra original debe ser considerada como la misma obra. O'Neill y Vizine-Goetz acompañan a sus definiciones de entidades bibliográficas de pautas para su eventual aplicación de forma consistente.

Svenonius (1992, p. 6), por su parte, ha señalado que las entidades bibliográficas son conjuntos de documentos. Sin embargo, la descripción de esta entidad, tal como se registra en un registro bibliográfico, no es literalmente una descripción de un conjunto de documentos sino más bien una descripción de un solo documento del conjunto. Este documento único se toma "para ser emblemático de cada documento del conjunto". Los atributos del documento, al ser mecanografiados, son vistos como atributos de la entidad bibliográfica formada por los documentos. Por tanto, hablamos del autor de una edición o el título de una edición y los registramos como elementos de datos en el registro bibliográfico de una edición.

Puede considerarse, entonces, que cada entidad bibliográfica está compuesta de atributos. En la medida que tienen varios atributos, participan de una variedad de relaciones entidad-atributo. Cada atributo considerado de uso bibliográfico se convierte en un elemento de dato individual en el registro bibliográfico para la entidad.

Las entidades bibliográficas están relacionadas con entidades de carácter nobibliográfico en virtud de un atributo relacionado. Las entidades bibliográficas pueden relacionarse también con otras entidades bibliográficas. Un ejemplo importante es la relación entre dos entidades que representan manifestaciones de la misma obra. Otros ejemplos, mencionados por Svenonius (1992, p. 7), incluyen la relación conjunto-subconjunto (jerárquica), la relación vertical (todo-parte), la relación cronológica (ediciones sucesivas) y la relación derivativa. En la figura 2 puede verse un ejemplo de entidades y relaciones bibliográficas.

\section{Hacia una taxonomía de las relaciones bibliográficas en el catá- logo de la biblioteca}

Una biblioteca representa una colección de documentos discretos que están relacionados entre sí de distintas formas. Algunos documentos, como las diferentes ediciones de una obra, están relacionados muy estrechamente e incluso pueden ser tratados como idénticos desde el punto de vista bibliográfico. Otras obras pueden estar relacionadas en mayor o menor grado (Van Houten, 1981, p. 369-370).

Una relación bibliográfica es una asociación entre dos o más obras o documentos bibliográficos. Algunos ejemplos son las circunstancias de publicación que vinculan dos o más documentos bibliográficos, como la atribución al mismo 


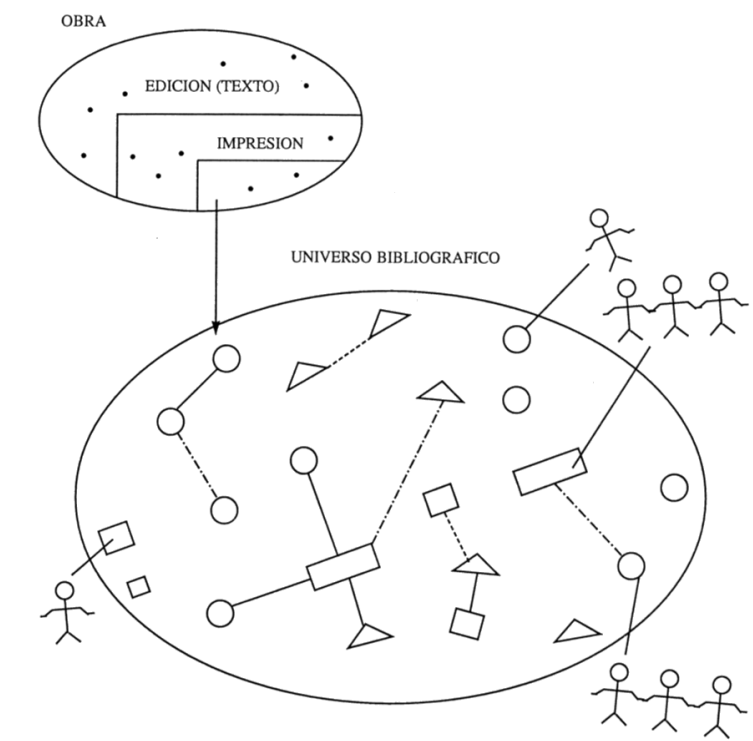

Obras en soporte material de distinto tipo, por ejemp., Textos, Películas, Registros Sonoros.

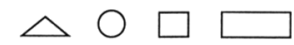

Diferentes tipos de Relaciones Bibliograficas, por ejemp., Cronologicas, Verticales, Derivativas.

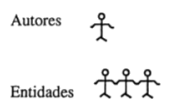

Fig. 2. Modelo parcial de entidades y relaciones bibliográficas.

autor, contener una variación de la misma obra, o documentos que forman parte de la misma serie. Teóricamente, las relaciones bibliográficas pueden incluir la asociación en virtud de características compartidas, como el idioma, el lugar de publicación, el editor y los aspectos físicos del tamaño, color de la encuadernación, etc. Aunque son significativos bibliográficamente para la descripción de los documentos, estos aspectos no se han utilizado para vincular registros bibliográficos en las anteriores reglas de catalogación. Sin embargo, estamos empezando a observar cómo se usan tales características para limitar las búsquedas en los catálogos en línea. Como señala Tillett (1991b, p. 150-151), "puede ser que todas las características comunes sean potencialmente útiles en el entorno automatizado". 
Al considerar los elementos esenciales de un registro bibliográfico, Weintraub (1979, p. 392) ha indicado que el primer problema es determinar las funciones de un sistema bibliográfico. La autora señala cuatro: la "función de identificación o lista de búsqueda, la función de reunión, la función de colocación y la función de selección o evaluación". Para Weintraub, la función de reunión permite encontrar siempre los mismos documentos bajo los mismos encabezamientos, y la función de colocación "consiste en agrupar los encabezamientos relacionados en un grupo". Su función de reunión es similar a la posibilidad del ordenador de recuperar publicaciones a través de una cierta estrategia de búsqueda y su función de colocación, a la búsqueda en un tesauro de términos para elegir el pertinente para una búsqueda. Pero la función de colocación discutida por Lubetzky (1979, p. 155) es mucho más sofisticada. Sirve para ordenar los asientos del catálogo y ofrece "un modelo de integración de los asientos diseñado para revelar al usuario del catálogo las relaciones intrínsecas de los materiales en la biblioteca y, por tanto, ayudarle a utilizar los recursos de la biblioteca de forma más completa". Obviamente esta función requiere siempre la agrupación de los registros bibliográficos asociados para su presentación. La agrupación puede llevarse a cabo a través de cualquier característica común compartida por dos o más documentos o a través de otros tipos de relaciones bibliográficas.

Para que un catálogo automatizado agrupe documentos relacionados, las obras que componen la colección deben ser identificadas sistemáticamente dentro de la base de datos catalográfica. Cuando un ordenador recupera documentos de acuerdo a una estrategia de búsqueda específica, separa la colección en dos grupos distintos: el que satisface la búsqueda y el que no lo hace. Si la búsqueda es muy específica y sólo se recuperan unos pocos documentos, puede no ser necesario ordenar esos documentos posteriormente y solamente viendo los datos bibliográficos pueden elegirse los documentos deseados. Estos documentos recuperados, sin embargo, no tienen que carecer necesariamente de relaciones con los documentos no recuperados. Una estrategia de búsqueda más general recuperará algunos de estos documentos, pero como la estrategia es genérica, las relaciones entre dos documentos determinados pueden ser muy débiles. Los documentos recuperados deben ser mostrados para representar las interrelaciones variables entre ellos (Van Houten, 1981, p. 370).

El estudio de las relaciones bibliográficas es normalmente una etapa necesaria en el proceso de crítica textual. Puesto que los catalogadores intentan informar a los usuarios sobre el contenido intelectual de las publicaciones, no solamente sobre su manifestación física, también realizan esta función aunque sea en un grado limitado. Sólo un catalogador de libros raros practica investigación original, otros copian la información relevante que encuentran en las fuentes existentes, a menudo internas, del documento que está siendo catalogado. En opinión

Scire. $2: 2$ (jul..-dic. 1996) 53-77 
de Hagler (1991, p. 46), "como en cualquier obra de detectives, la habilidad reside no tanto en localizar la información requerida como en conocer lo que se busca". Las relaciones más complejas son materias para investigaciones extensas, normalmente por especialistas en la materia más que por bibliógrafos per se, por ejemplo, alguien consulta un estudio de literatura comparada, no una bibliografía, para descubrir los vínculos entre las distintas leyendas medievales de Tristán. Sin embargo, registrar los textos sucesivos de Moby Dick es básicamente un trabajo bibliográfico si sus resultados se detallan en un volumen grueso de prosa o se esbozan en unas pocas palabras en una escueta ficha de catálogo para cada variante localizada.

Las relaciones bibliográficas mencionadas en muchas citas y registros catalográficos más comunes, importantes y fáciles de expresar se revelan normalmente dentro del propio documento. Hagler ha señalado que existen relaciones entre:

1. Ediciones de la misma obra,

2. Segundas partes, continuaciones, etc.,

3. Documentos independientes física y bibliográficamente pero publicados y concebidos para ser usados juntos,

4. Obras importantes identificables independientemente pero contenidas en una publicación, y

5. Documentos en la misma serie (ibidem, p. 47).

Por su propia naturaleza, algunas relaciones son más tenues y subjetivas que otras. Por ejemplo, es mucho más fácil juzgar si una obra es una traducción que si es una parodia (Holley, 1992, p. 61). Además, las relaciones bibliográficas pueden ser multidimensionales. Por ejemplo, la segunda edición de una obra puede ser publicada en una serie diferente distinta de la primera edición, una reimpresión de un registro sonoro de rock puede contener un sonido nuevo reemplazando la banda sonora del registro original y una colección de microformas reúne a menudo material de varias publicaciones (Hagler, 1991, p. 95).

Aunque las relaciones bibliográficas se incorporaron a los catálogos hace más de un siglo, hasta mediados de los años 70 no han sido objeto de un examen formal. El desarrollo de los catálogos en línea y la aplicación de las AACR2 han provocado una demanda de más información, teórica y empírica, sobre la base del diseño de los catálogos y las futuras reglas de catalogación.

Los datos existentes consisten en los hallazgos de unos pocos estudios empíricos dirigidos a examinar los principios básicos de la catalogación descriptiva y algunos tipos de relaciones de equivalencia de nombres, incluyendo categorizaciones de variaciones de nombres y una propuesta taxonómica para las relaciones 
entre los nombres (Tillett, 1991b, p. 151-152). Sin embargo, no ha habido datos empíricos accesibles previamente para las relaciones bibliográficas.

\subsection{UNIMARC}

Seguramente las primeras obras significativas sobre las relaciones bibliográficas son las definiciones de relaciones en el formato UNIMARC (1977 y 1980) y el análisis de las relaciones jerárquicas llevado a cabo por Goossens y MazurRzesos. Las definiciones de relaciones bibliográficas que aparecían en el UNIMARC Format (1980, p. 58-59) sugieren una estructura filosófica para las relaciones bibliográficas categorizándolas en los tres tipos siguientes:

1. Verticales: son las relaciones jerárquicas del todo con sus partes y de las partes con el conjunto, por ejemplo, vínculos descendentes (una publicación seriada con sus subseries o con los volúmenes individuales de la serie) o vínculos ascendentes (el volumen individual con su subserie y/o serie).

2. Horizontales: son las relaciones entre versiones de un documento en diferentes idiomas, formatos, soportes, etc.

3. Cronológicas: son las relaciones en el tiempo entre fascículos de un documento, por ejemplo, la relación de una publicación seriada con sus predecesores y sucesores.

Los principios taxonómicos requieren, según Tillett (1991b, p. 153), categorías mutuamente exclusivas y totalmente exhaustivas y las tres categorías anteriores no lo son. No son exhaustivas porque omiten algunas relaciones, como las existentes entre copias, entre un suplemento y la obra a la que acompaña, entre un registro y la obra que analiza o entre la revisión de un libro y la obra revisada. Se ha sugerido que las categorías UNIMARC pueden generalizarse para incluir estas relaciones pero esta generalización "ofusca diferencias importantes en los tipos de relaciones".

Por ejemplo, la definición de relación vertical de UNIMARC puede ser replanteada como vínculos bidireccionales entre un conjunto y sus partes. Si generalizamos para incluir todas las relaciones jerárquicas, como han hecho Goossens y Mazur-Rzesos (como describiremos más adelante), podemos incluir los componentes de un conjunto más grande y las obras distintivas donde un documento es predominante y otro subordinado. Pero estas son realmente tipos de relaciones muy diferentes y el hecho de que se utilice un único tipo de diagrama jerárquico para representarlas no las hace idénticas.

La tercera edición del UNIMARC (1987) siguió manteniendo los tres tipos de relaciones bibliográficas de las ediciones anteriores. En un taller celebrado en Sidney en 1989 para abordar el estado de la cuestión del formato, Cristine 
Bossmeyer presentó un trabajo, "particularmente importante" en opinión de Agenjo Bullón (1993, p. 21), sobre las técnicas de vinculación en UNIMARC. Tras exponer la estructura de los vínculos accesibles en el formato, Bossmeyer (1989, p. 21) consideró la técnica de vinculación desde un punto de vista bibliográfico. Aunque señala que las tres categorías de relaciones bibliográficas (verticales, horizontales y cronológicas) son las que se usan más comúnmente en los sistemas de catalogación, reconoce que "hay otras relaciones utilizadas en los sistemas catalográficos y descritas en la literatura que no pueden ser cubiertas por UNIMARC".

Por otro lado, las tres categorías UNIMARC tampoco son mutuamente exclusivas. La distinción entre la horizontal y la cronológica es vaga, como en el caso de un manual publicado anualmente en versiones actualizadas con pequeños cambios de título. La relación entre las ediciones consecutivas es tanto horizontal, en tanto aparece en versiones, como cronológica, en tanto se publica anualmente con títulos sucesivamente diferentes. En definitiva, Tillett (1991b, p. 154) piensa que las tres categorías desarrolladas para el formato UNIMARC son indudablemente un paso importante en el proceso de la descripción de las relaciones bibliográficas pero adolecen de los problemas mencionados.

\subsection{Las relaciones jerárquicas de Goossens y Mazur-Rzesos}

El artículo sobre las relaciones jerárquicas publicado por Goossens y MazurRzesos (1982) no cita las definiciones del primer UNIMARC, sino otras derivadas de las deliberaciones del comité de la IFLA que desarrolló las definiciones, en las cuales participó Goossens (1).

Goossens y Mazur-Rzesos introdujeron una representación esquemática de las relaciones jerárquicas para expresarlas de forma sencilla y superpuesta por medio de estructuras arborescentes complejas. Su objetivo es suministrar la base teórica para soluciones manuales y automatizadas que expresen tales relaciones. Los autores señalan que los ejemplos prácticos se limitaron a estructuras arborescentes con un máximo de tres niveles, planteando la cuestión de la posible necesidad de un mayor número de niveles en los catálogos. Los autores demostraron con éxito la aplicación de estructuras arborescentes jerárquicas para ciertos tipos de materiales bibliográficos: publicaciones periódicas complejas, monografías complejas, series, suplementos, material de acompañamiento y descripción analítica. En conreto, la jerarquía con el nivel más alto es denominada nivel de conjunto, los niveles intermedios son llamadas niveles de subconjunto y el nivel más bajo es denominado nivel de pieza, utilizando notaciones especiales para cada nivel.

Este esquema funciona muy bien para las jerarquías verdaderas, como las series y las subseries, pero la estructura arborescente no funciona tan bien cuan-

Scire. 2 : 2 (jul.-dic. 1996) 53-77 
do expresa partes suplementarias o acompañantes. Las estructuras arborescentes para suplementos y obras acompañantes identifican relaciones que no son definidas jerárquicamente (Tillett, 1991b, p. 154).

Aparte del análisis en profundidad de las relaciones jerárquicas de Goossens y Mazur-Rzesos, no se ha llevado a cabo otro análisis exhaustivo de las relaciones bibliográficas hasta el estudio realizado por Tillett.

\subsection{Las clases de documentos bibliográficos de McCallum}

McCallum (1982, p. 282), en un artículo donde presentaba la técnica utilizada por los formatos MARC para codificar los registros bibliográficos de partes componentes de documentos, señaló que los campos que vinculan asientos se usan cuando se requieren referencias a los documentos bibliográficos que contienen relaciones específicas al documento al que se envía. La autora describía dos clases de documentos bibliográficos:

1. Los documentos relacionados que pueden ayudar al usuario a continuar la búsqueda pero no son requeridos necesariamente para obtener el documento al que se envía, como asientos anteriores o traducciones del documento al que se envía.

2. Los documentos relacionados que son requeridos para obtener el documento al que se envía, como los documentos fuente para las partes componentes.

McCallum no desarrolló esta clasificación funcional. Lo que sugiere la autora es una clasificación dicotómica de las relaciones en documentos "en contenedores" y documentos contenidos independientemente. Los documentos "en contenedores" son partes componentes que tienen un documento fuente y la persona que lleva a cabo la búsqueda debe tener conocimiento del documento fuente para obtener físicamente el componente. Los documentos contenidos de forma separada son los que presentan otros tipos de relaciones. Esta categorización, como señala Tillett, abarca además otras relaciones distintas de las bibliográficas, puede concebirse la inclusión de la relación de los puntos de acceso con los registros bibliográficos y las relaciones entre las variaciones de los nombres y entre los nombres relacionados (1991b, p. 154).

\subsection{La propuesta de relaciones bibliográficas de Tillett}

El estudio analítico de las relaciones jerárquicas de Goossens y MazurRzesos había comenzado a examinar las relaciones bibliográficas de manera rigurosa. Parecía evidente, pues, la necesidad de estudios adicionales basados en estudios empíricos sobre la naturaleza y la extensión de las relaciones bibliográficas en los catálogos de las bibliotecas. Atendiendo a estas necesidades, Tillett 
llevó a cabo un estudio empírico durante los años 1984-1986, que constituyó su disertación doctoral.

La autora ha desarrollado una taxonomía que "puede ser vista como una expansión de la división de McCallum y una expansión y clarificación de las categorizaciones del UNIMARC original" (Tillett, 1988, p. 5). Los siete tipos de relaciones bibliográficas derivadas de dicho estudio son los siguientes:

\subsubsection{Relaciones de equivalencia}

Las relaciones de equivalencia se dan entre copias exactas de la misma manifestación de una obra, o entre un documento original y sus reproducciones, siempre que se preserve el contenido intelectual y artístico y la autoría. Se incluyen aquí las copias, fascículos, facsímiles y reimpresiones, fotocopias, microformas y otras reproducciones similares (Tillett, 1991b, p. 156).

Las reglas de catalogación han contemplado históricamente seis tipos de instrumentos de enlace para indicar los documentos equivalentes en los registros bibliográficos:

1. Un asiento "con guión" para el documento equivalente dentro del registro del documento original (2).

2. Una nota en el registro bibliográfico del documento original reconociendo el documento equivalente.

3. Una nota en el registro bibliográfico del documento equivalente reconociendo el original.

4. Notas para vincular registros bibliográficos independientes para el original y los documentos relacionados.

5. El mismo encabezamiento de título uniforme usado en los registros del documento equivalente y el original.

6. Una anotación de fondos y localizaciones sobre el documento equivalente en el registro bibliográfico para el original o en el catálogo topográfico para el original.

Los instrumentos de enlace de las notas y los títulos uniformes se usan para relacionar publicaciones en el universo bibliográfico en general, mientras los instrumentos de enlace de los asientos "con guión" y anotaciones de fondos se usan para relacionar los fondos concretos de una biblioteca determinada (Tillett, 1991a, p. 395). 


\subsubsection{Relaciones derivativas}

Las relaciones derivativas, denominadas relaciones horizontales en UNIMARC, se dan entre un documento bibliográfico y una modificación basada en el mismo documento. Esto incluye:

- Variaciones o versiones de otra obra, como ediciones, revisiones, traducciones, sumarios, resúmenes, versiones condensadas

- Adaptaciones o modificaciones que han producido obras nuevas pero que están basadas en obras anteriores

- Cambios de género, como dramatizaciones o novelizaciones

- Nuevas obras basadas en el estilo o el contenido temático de otras obras, como traducciones libres, paráfrasis, imitaciones y parodias (Tillett, 1991b, p. 156).

Las relaciones derivativas abarcan toda la gama de relaciones que van de las casi idénticas a las totalmente distintas. La conexión global entre estas relaciones diversas es el hecho de que existe alguna obra original de la cual se deriva otra obra, ya sea en el contenido intelectual, el estilo o el contenido temático. Considerando la diversidad de las relaciones de esta categoría, no es sorprendente que se utilice una diversidad similar de instrumentos de enlace para expresarlas en un catálogo. Se incluyen aquí las referencias, los asientos "con guión" para ediciones secundarias, menciones de edición, notas, títulos uniformes, encabezamientos de materia, encabezamientos principales comunes, títulos de ordenación y encabezamientos secundarios. No existe una regla racional para preferir un instrumento concreto sobre otro para vincular los documentos bibliográficos y sus derivaciones y, por tanto, las reglas son a menudo inconsistentes (Tillett, 1991a, p. 297-398).

\subsubsection{Relaciones descriptivas}

Las relaciones descriptivas se dan entre una obra o documento bibliográfico y una descripción, crítica, evaluación o revisión de dicha obra, como la existente entre un documento y una revisión que lo describe. También se incluyen las ediciones anotadas, comentarios, críticas, etc (Tillett, 1991b, p. 156).

Los instrumentos de enlace para las relaciones descriptivas incluyen notas sobre el documento descrito en los asientos analíticos para la "descripción", encabezamientos principales comunes, notas sobre la descripción y asientos secundarios o asientos de materias para la obra original. Cuando una descripción se publica siempre con el documento que describe, las reglas de catalogación ponen el acento en la catalogación del documento descrito con una nota sobre la descripción. Cuando la descripción es predominante o se publica de forma separada, las reglas de catalogación usan notas y asientos secundarios o asientos de 
materias para vincular la descripción con el documento descrito. En raras ocasiones (incunables, manuscritos y mapas, fundamentalmente), el documento descriptivo se anota en el registro bibliográfico del documento descrito como una fuente de referencia (Tillett, 1991a, p. 398).

\subsubsection{Relaciones todo-parte (o parte-todo)}

Las relaciones todo-parte (o parte-todo), denominadas relaciones verticales en UNIMARC o relaciones jerárquicas por Goossens y Mazur-Rzesos, se dan entre una parte componente de una obra o documento bibliográfico y su conjunto, como ocurre con una selección individual y la antología, colección o serie global (Tillett, 1991b, p. 156).

Una amplia variedad de instrumentos de enlace han sido utilizados para representar las relaciones todo-parte dentro de los registros bibliográficos, dependiendo del tipo de relación todo-parte expresada: relaciones de "contiene", relaciones extractivas o relaciones de resumen. Los instrumentos incluyen:

- Notas de contenido indicando partes específicas.

- Asientos “con guión” para copias de partes eliminadas.

- Asientos analíticos para las partes.

- Asientos secundarios para la obra global o la parte.

- Descripciones a varios niveles y asientos “con guión” para incorporar todos los componentes dentro de una descripción bibliográfica.

- Encabezamientos de título uniforme para la obra más grande actuando como encabezamientos de asiento principal para las partes.

- Referencias explicativas identificando las partes de una obra.

Esta variedad ilustra la diversidad en el tratamiento catalográfico de las partes de un conjunto. El método más simple para mostrar la relación todo-parte es describir la obra más grande e indicar de alguna manera sus contenidos; así, se elabora un registro para el conjunto y sus partes. Sin embargo, cuando las partes requieren descripción adicional a la que suministra el registro del conjunto, se elaboran registros múltiples, de forma que se dé cada parte en un registro bibliográfico independiente citando el conjunto (Tillett, 1991a, p. 400).

\subsubsection{Relaciones de acompañamiento}

Las relaciones de acompañamiento se dan entre un documento bibliográfico y otro que lo acompaña como, por ejemplo, dos documentos que se complementan en la misma medida uno a otro o uno que amplía al documento principal o predominante. Hay ejemplos de relaciones entre documentos y sus materiales acompañantes donde un documento es predominante y el otro subordinado, como un texto y sus suplementos; o un documento que suministra acceso a otro

Scire. 2 : 2 (jul.-dic. 1996) 53-77 
documento, como las concordancias, índices, catálogos de bibliotecas, etc.; o documentos de similar estatus pero que no tienen una ordenación cronológica específica, como es el caso de las partes de un conjunto documental (Tillett, 1991b, p. 156).

Los códigos de catalogación han incorporado siempre reglas para los materiales acompañantes. Los dispositivos usados para expresar las relaciones de acompañamiento son:

- Material anejo en la descripción física.

- Notas.

- Asiento "con guión”.

- Descripción a varios niveles.

- Registros separados con notas de enlace.

Excepto el asiento "con guión", todos ellos han sobrevivido en los actuales códigos catalográficos. Estos asientos "con guión" eran utilizados en vez de un registro separado o una descripción a varios niveles (1991a, p. 401).

\subsubsection{Relaciones secuenciales}

Las relaciones secuenciales, denominadas relaciones cronológicas en UNIMARC, se dan entre documentos bibliográficos que continúan o preceden a otro, como ocurre entre los títulos sucesivos de una publicación seriada, las continuaciones de una monografía o entre las distintas partes de una serie numerada (Tillett, 1991b, p. 156).

Los instrumentos usados para las relaciones secuenciales son:

- Notas de los primeros títulos.

- Notas de los últimos títulos.

- Notas de los títulos inmediatamente precedentes o sucesivos.

- Agrupación de asientos secundarias.

- Títulos uniformes.

El asiento de títulos sucesivos con asientos secundarios que los vinculen con los títulos precedentes y sucesivos es el método preferido normalmente para enlazar secuencialmente documentos relacionados (1991a, p. 401).

\subsubsection{Relaciones de características compartidas.}

Las relaciones de características compartidas se dan entre un documento bibliográfico y otros que no están relacionados de ninguna forma pero tienen en común el autor, el título, la materia u otras características utilizadas como punto de acceso en un catálogo, como un idioma, fecha de publicación o país de publi- 
cación (Tillett, 1991b, p. 156).

Este tipo de relaciones son las más generales de todas, porque se dan siempre que un punto de acceso se encuentra duplicado en un fichero determinado (Tillett, 1991a, p. 402).

\section{Estudio de un caso concreto: las relaciones bibliográficas en el catálogo de la Library of Congress}

\subsection{Objetivos del estudio}

Tillett (1992) llevó a cabo un estudio empírico en 1986 para examinar la extensión de las relaciones bibliográficas reflejada en la frecuencia de su ocurrencia dentro de la base de datos automatizada (1968-julio 1986) de la Library of Congress. La frecuencia de la ocurrencia fue determinada por el recuento de las incidencias de los códigos específicos asociados con cada tipo de relación en los registros automatizados. También examinó algunas características de los documentos bibliográficos que exhibían relaciones concretas (la lengua, el lugar de publicación, la fecha de publicación, la materia y el formato bibliográfico) ya que pensaba que dichos factores podían ser útiles para predecir tipos concretos de relaciones en los futuros sistemas de catalogación. Esta información puede ser de utilidad potencial para quienes toman decisiones y para los diseñadores de sistemas en la evaluación de métodos adecuados para diseñar relaciones específicas en los catálogos y las reglas de catalogación del futuro.

\subsection{Metodología}

Se revisaron los formatos MARC para seleccionar campos que contuviesen información explícita sobre las relaciones bibliográficas (aparte de los campos 500 de notas generales). El resultado fue una lista de etiquetas, indicadores, códigos de subcampo y valores que totalizaban 134 relaciones bibliográficas codificadas específicamente. Cada relación fue asociada a una categoría taxonómica apropiada: equivalencia, derivativa, descriptiva, todo-parte, acompañamiento, secuencial y característica compartida. Para la categoría de relaciones descriptivas no existían otros códigos MARC específicos además de las notas generales del campo 500. La categoría de relaciones de características compartidas, por otro lado, podía ser indicada casi por cualquier código MARC y fue eliminada del estudio empírico debido a la complejidad de su recuento. No se encontraron registros para 14 de las 134 relaciones codificadas. Los restantes 120 códigos de relaciones fueron traducidos a preguntas automatizadas para recuperar registros de la base de datos. Dado que la LC tiene para su propia gestión de rutina de la base de datos el sistema JANUS de programas automatizados, que cuenta la frecuencia de la ocurrencia de campos y subcampos específicos para cada uno de sus ficheros MARC, fue posible realizar las preguntas para determinar el núme-

Scire. $2: 2$ (jul.-dic. 1996) 53-77 
ro de ocurrencias de cada relación para cada uno de los factores específicos del estudio (ibidem, p. 165-166).

\subsection{Resultados: datos empíricos sobre las relaciones bibliográficas}

El estudio puso de manifiesto que muchos registros que contienen relaciones bibliográficas pueden ser clasificados como registros de documentos en inglés publicados en Estados Unidos entre 1970 y 1979. Una excepción es el material implicado en las relaciones de equivalencia, que fue publicado predominantemente en los años 80. Para los libros, mapas y materiales visuales (los únicos formatos con datos de materia), la ciencia fue la materia prioritaria asociada con las relaciones de acompañamiento, las ciencias sociales con las relaciones de todoparte, las humanidades con las relaciones derivativas, y la categoría de materia desconocida con las relaciones de equivalencia. El formato para el mayor número de registros para las relaciones de equivalencia, derivativas y todo-parte fue el de los libros, mientras que los formatos que exhibían relaciones de acompañamiento o secuenciales con el mayor número de registros eran los materiales visuales y las publicaciones seriadas, respectivamente. El número de registros de mapas para las relaciones todo-parte y derivativas fue muy superior al esperado de la distribución de los mapas en la base de datos de la LC en su conjunto.

De los datos recogidos en el estudio de los códigos MARC únicos, Tillett encontró los siguientes factores que caracterizaban a los registros que contenían información para cada tipo de relación:

- Registros con relaciones de equivalencia: el 63,4\% eran de libros (el 78\% de lo que podía esperarse si los formatos hubiesen estado distribuidos de forma similar en el fichero de relaciones y en la base de datos de la LC en su conjunto), el 82,88\% eran de la categoría de materia "desconocida", el $13,05 \%$ eran de humanidades, el 68,88\% eran de lengua inglesa, el 40,46\% estaban publicados en Estados Unidos y el 30,44\% habían sido publicados en los años 80 .

- Registros con relaciones derivativas: el 79,03\% eran de libros (alrededor del 3\% menos del porcentaje de registros para libros que se esperaba encontrar en la base de datos de la LC en su conjunto), el 46,73\% eran de humanidades, el 80,74\% eran de lengua inglesa, el 52,41\% estaban publi cados en Estados Unidos y el 46,56\% habían sido publicados en los años 70.

- Registros con relaciones descriptivas: fueron caracterizados por dos incidencias encontradas en el estudio del campo 500 de notas generales y eran de libros de humanidades en lengua inglesa, publicados en Estados Unidos; uno fue publicado en 1975 y otro en 1980. 
- Registros con relaciones todo-parte: el 75,01\% eran de libros (un 8\% menos del porcentaje de registros encontrado para libros en la base de datos de la LC en su conjunto), el 41,70\% eran de ciencias sociales, el 56,99\% eran de lengua inglesa, el 38,72\% estaban publicados en Estados Unidos y el 55,37\% habían sido publicados en los años 70 .

- Registros con relaciones de acompañamiento: el 65,98\% eran de materiales visuales (un asombroso porcentaje 24 veces mayor de registros para materiales visuales del esperado en la base de datos de la LC en su conjunto), el 39,44\% eran de ciencias, el 81,30\% eran de lengua inglesa, el $79,68 \%$ estaban publicados en Estados Unidos y el 49,66\% habían sido publicados en las años 70 .

- Registros con relaciones secuenciales: si se añaden los datos del estudio del campo 500 de notas generales, el 99,46\% de los registros con información de relaciones secuenciales son de publicaciones seriadas (un $880 \%$ más que la distribución de publicaciones seriadas en la base de datos de la LC en su conjunto). En el estudio de los códigos MARC explícitos, Tillett encontró que el 79,42\% de los registros con relaciones secuenciales eran de documentos en lengua inglesa, el 57,74\% estaban publicados en Estados Unidos y el 25,34\% habían sido publicados en los años 70. No existían datos de materias accesibles para las relaciones secuenciales. Otro 0,54\% de los registros con información sobre relaciones secuenciales eran registros de libros, encontrados a través del estudio del campo 500 de notas generales (ibidem, p. 183-184).

- Registros con relaciones de características compartidas: este tipo de relaciones no fueron estudiadas por Tillett. Sin embargo, existen datos de un estudio realizado unos años antes por McCallum y Godwin (1981), quienes analizaron los encabezamientos duplicados en cuatro ficheros de registros MARC de la LC (libros, publicaciones seriadas, mapas y películas) que contenían versiones mejoradas de todos los registros que habían sido distribuidos desde 1969 hasta octubre de 1979. Se procesaron un total de 1.336.182 registros bibliográficos, incluyendo 1.134 .069 del fichero de libros, 90.174 del fichero de publicaciones seriadas, 60.758 del fichero de mapas y 51.176 del fichero de películas. Los resultados mostraron que sólo 633.048 de los 1.308 .193 encabezamientos de nombres personales que aparecieron en los registros (el 48\% de éstos) eran distintos. El 52\% restante duplicados de encabezamientos distintos. De forma similar, el $26 \%$ de los nombres de entidades eran distintos, siendo duplicados el $74 \%$, y el $71 \%$ de los nombres de congresos eran distintos, con sólo el $29 \%$ de duplicados (ibidem, p. 197). 


\section{2}

Los autores también contabilizaron la frecuencia de la ocurrencia de los encabezamientos de nombres en los ficheros bibliográficos: el 66\% de nombres personales distintos, el $62 \%$ de nombres de entidades distintos y el $84 \%$ de nombres de entidades distintos ocurrían sólo una vez en los catálogos. El porcentaje de nombres corporativos con ocurrencias únicas era sorprendentemente cercano al de nombres personales pero, sin embargo, el porcentaje de nombres que tenían ocurrencias múltiples cae más lentamente para los nombres corporativos que para los personales. Mientras el 5,47\% de los nombres corporativos ocurrían 10 o más veces, sólo el 1,92\% de los nombres personales ocurrían 10 o más veces (ibidem, p. 198).

Básicamente tenemos información sobre las características de los documentos bibliográficos que presentan relaciones e información sobre el formato MARC relativa a las relaciones. Estos datos nos indican que factores como la lengua, el país de publicación y la fecha de publicación caracterizan a los documentos que participan en las relaciones bibliográficas (es decir, en el fichero de relaciones) de forma muy parecida a los factores que caracterizan a los documentos en la base de datos de la LC en su conjunto. Sin embargo, para el factor de los formatos MARC existe una disimilitud. En el fichero de relaciones hay un número mucho mayor de registros de materiales visuales para las relaciones de acompañamiento, de registros de mapas para las relaciones derivativas y todo-parte y de registros de publicaciones seriadas para las relaciones secuenciales que en la base de datos de la LC. Vemos, por tanto, que las relaciones de acompañamiento se dan más a menudo entre materiales visuales, las relaciones secuenciales entre las publicaciones seriadas y las relaciones de todo-parte, derivativas y de equivalencia entre los libros.

Por otro lado, al examinar la posibilidad de predecir el tipo de relación del formato, entontramos que los libros, los mapas, los materiales visuales y los documentos musicales muestran más relaciones todo-parte que cualquier otro $(79,24 \%, 82,10 \%, 51,55 \%$ y $55,6 \%$ respectivamente), mientras las publicaciones seriadas muestran relaciones secuenciales más frecuentemente $(73,35 \%)$.

Si se hace la predicción del idioma, los materiales en todas las lenguas muestran más a menudo relaciones todo-parte $(54,35 \%$ de los materiales en lengua inglesa, 72,67\% de lengua francesa, 79,08\% de lengua alemana, $89,18 \%$ de lengua italiana, $80,82 \%$ de lengua portuguesa, $68,54 \%$ de lengua rusa y $84,32 \%$ de lengua española). 
Del mismo modo, las relaciones todo-parte son las que se dan más a menudo por país de publicación, excepto para publicaciones canadienses, que presentan relaciones secuenciales con más frecuencia.

Por fecha de publicación, las relaciones todo-parte ocurren más a menudo, excepto para fechas anteriores a 1700, donde las relaciones derivativas se dan con más frecuencia (45,74\% de todos los tipos de relaciones) y para $1800-1899$ y 1910-1949, donde las relaciones secuenciales se producen más a menudo (Tillett, 1992, p. 184).

Para dar una idea del número total de registros que muestran relaciones bibliográficas en la base de datos LC MARC de 1968-julio 1986, el número de registros contados en el estudio con relaciones codificados explícitamente debe añadirse al número de registros encontrados en el estudio del campo 500 de notas generales. Pero aun así no se puede tener un recuento completo porque no se han contado ninguna de las relaciones bibliográficas para las que no existe un código MARC ni tampoco las relaciones de características compartidas. De todas formas, el estudio ofreció un total estimado de 2.143.256 registros que contenían información de relación, que es el $75 \%$ del total de la base de datos. No obstante, debe señalarse que el método usado para contar la frecuencia de los registros permitía que un porcentaje indeterminado de registros estuviesen duplicados en los distintos tipos de relaciones, de forma que el número real de registros que contienen información de relación es menor del $75 \%$ de la base de datos total.

Respecto al número de registros que contienen información de relación hay algunos hallazgos interesantes. De los 1.254.000 registros que contienen información de las relaciones bibliográficas en el estudio de las relaciones codificadas explícitamente, 42.000 (3,35\%) eran para las relaciones de equivalencia (3), $179.000(14,17 \%)$ para las relaciones derivativas, $782.000(62,36 \%)$ para las relaciones todo-parte, $49.000(3,91 \%)$ para las relaciones de acompañamiento y $202.000(16,11 \%)$ para las relaciones secuenciales. Un futuro estudio debería ser realizado, en opinión de Tillett, para determinar la correlación cruzada de los factores para estimar de forma más precisa el porcentaje de una base de datos que refleja relaciones bibliográficas (Tillett, 1992, p. 184).

\subsection{Conclusiones}

A la vista de estos datos Tillett concluye que los registros bibliográficos actuales incluyen un grado de redundancia de la información bibliográfica. Esta redundancia toma la forma de registros de puntos de acceso secundarios. Si asumimos que el futuro formato del registro bibliográfico debe optimizar las capacidades de los programas automatizados, podemos reducir la redundancia existente en el formato MARC. Por ejemplo, los requerimientos de ordenación y presentación pueden ser incluidos en el registro de forma más elegante que la

Scire. $2: 2$ (jul.-dic. 1996) 53-77 
actual práctica de repetir la información que va a ser usada como puntos de acceso. Para las relaciones bibliográficas dentro del registro bibliográfico los factores importantes son: (1) una citación de identificación de los vínculos con otra obra relacionada y (2) la codificación explícita de otros mecanismos para identificar el tipo de relación (ibidem, p. 185). Además, como señala Attig (1989, p. 142), en los sistemas actuales, los datos de los campos vinculantes son raramente utilizados por los usuarios para establecer un vínculo directo entre los registros. En vez de eso, quien realiza la búsqueda espera usar la información sobre los documentos relacionados como la bases para búsquedas adicionales. En definitiva, los dispositivos exactos para el almacenamiento y transmisión de la información sobre relaciones bibliográficas en los formatos MARC u otros formatos bibliográficos del futuro son cuestiones que deben ser investigadas.

Finalmente, aunque la función del catálogo estableciendo relaciones y organizando los elementos de datos es sin duda muy importante, sería conveniente recordar, como indica Nwafor (1992, p. 78), que debe resistirse la tentación "de ignorar la existencia de excelentes índices y resúmenes [...] o, peor, elaborar el catálogo duplicando los contenidos de estas herramientas bibliográficas”.

\section{Notas.}

(1) Los autores dan las siguientes definiciones de las relaciones bibliográficas: “(1) las relaciones jerárquicas: los vínculos del conjunto con sus partes y de las partes con un conjunto (por ejemplo, la relación entre una serie y sus monografías); (2) las relaciones cronológicas: los vínculos en el tiempo entre los fascículos que aparecen de un documento (por ejemplo, la relación de una publicación periódica con sus predecesoras y sus sucesoras); (3) las relaciones horizontales: los vínculos de versiones de un documento (por ejemplo, en diferentes idiomas, ediciones locales, reediciones, formatos distintos, etc.)" (Goessens y Mazur-Rzesos, 1982, p. 14).

(2) Un asiento con guión es un asiento subordinado a otro bajo el que aparece. Estos asientos se encontraban ya en los primeros catálogos y fueron abolidos en 1978 por las AACR2 al prescribir asientos separados para documentos que podían haber tenido un asiento con guión bajo las reglas anteriores o al permitir la descripción a varios niveles.

(3) A Grimaldi (1993, p. 136) le parece muy significativa la baja tasa de frecuencia de las relaciones de equivalencia. Este fenómeno parece derivarse de la asunción del criterio tipográfico-editorial para valorar la definición de la naturaleza de la entidad bibliográfica y de sus relaciones, con el consiguiente ocultamiento de un gran número de casos de equivalencia textual. En consecuencia, se reconocen como equivalentes las copias y las impresiones sucesivas de una publicación, pero son consideradas como nuevas ediciones algunos tipos de emisiones o de copias o las reproducciones facsimilares de obras efectuadas a través de la recomposición tipográfica del texto original. 


\section{Referencias}

Agenjo Bullón, X. (1993). El formato UNIMARC: historia, estado actual y perspectivas de futuro. // Boletín de la ANABAD. 43 : 2 (1993) 19-23.

Amat, C. B. ; Pérez Salinas, I.; Ramos Pérez, A. (1989). Aplicación del modelo entidadrelación a la definición de datos y al establecimiento de relaciones en el diseño de una base de datos de información de actualidad. // Item. 5 (1989) 63-81.

Attig, J. C. (1989). Descriptive cataloging rules and machine readable record structures: some directions for parallel development. // The conceptual foundations of descriptive cataloging. Edited by Elaine Svenonius. San Diego [etc.] : Academic Press, 1989. p. 135-148.

Bossmeyer, C. (1989). Linking techniques in UNIMARC. // UNIMARC Workshop (1988, Sidney). UNIMARC in theory and practice : papers form the UNIMARC Workshop, Sidney, Australia, August 1988. Edited by Sally H. McCallum and Winston D. Roberts. London, IFLA Universal Bibliographic Control and International MARC Programme, 1989. p. 19-30.

Fidel, R. ; Crandall, M. (1988). The AACR2 as a design schema for bibliographic databases. // Library Quarterly. 58 : 2 (1988) 123-142.

Goessens, P. ; Mazur-Rzesos, E. (1982). Hierarchical relationships in bibliographic descriptions: problem analysis. // Hierarchical relationship in bibliographical descriptions: INTERMARC Software Subgroup Seminar 4. Essen : Gesamthochschulbibliothek Essen, 1982. p. 13-26.

Griffiths, P. (1989). Entity-relationship analysis, the operational requirement and a buyer's view of the market for automated library systems. // Program. 23 : 1 (1989) 13-28.

Grimaldi, T. (1993). Catalogazione e ricerca dell'informazione (III). // Il bibliotecario. 38 (1993) 123-137.

Hagler, R. (1991). The bibliographic record and information technology. 2nd ed. Twickenham : Adamantine Press, 1991.

Holley, R. P. (1992). Panel discussion on first day's papers. // Seminar on Bibliographic Records (1990. Stockholm). Seminar on Bibliographic Records : proceedings of the seminar held in Stockholm, 15-16 August 1990, and sponsored by the IFLA UBCIM Programme and the IFLA Division of Bibliographic Control. Edited by Ross Bourne. München [etc.] : Saur, 1992. p. 61-67.

Koenig, M. E. D. (1985). Data relationships : bibliographic information retrieval systems and database management systems. // Information Technology and Libraries. $4: 3$ (1985) 247-272.

Leazer, G. H. (1992). An examination of data elements for bibliographic description : toward a conceptual schema for the USMARC format. // Library Resources \& Technical Services. $36: 2$ (1992) 189-208.

Lubetzky, S. (1979). The traditional ideals of cataloging and the new revision. // The nature and future of the catalog : proceedings of the ALA Information Science and Automation Division's 1975 and 1977 Institutes on the Catalog. Edited by Maurice J. Freedman and S. Michael Malinconio. Phoenix : Oryx Press, 1979. p. 153-169.

Scire. 2 : 2 (jul.-dic. 1996) 53-77 
Lubetzky, S. (1985). The objectives of the catalog. // Foundations of cataloging: a sourcebook. Edited by Michael Carpenter and Elaine Svenonius. Littleton, Col. : Libraries Unlimited, 1985. p. 189-191.

McCallum, S. H. (1982). MARC record-linking technique. // Information Technology and Libraries. $1: 3$ (1982) 281-291.

McCallum, S. H.; Godwin, J. L. (1981). Statistics on headings in the MARC file. // Journal of Library Automation. 14 : 3 (1981) 194-201.

Nwafor, B. U. (1992). Panel discussion on first day's papers. // Seminar on Bibliographic Records (1990. Stockholm). Seminar on Bibliographic Records: proceedings of the seminar held in Stockholm, 15-16 August 1990, and sponsored by the IFLA UBCIM Programme and the IFLA Division of Bibliographic Control. Edited by Ross Bourne. München [etc.]: Saur, 1992. p. 77-78.

O’Neill, E. T. ; Vizine-Goetz, D. (1989). Bibliographic relationships: implications for the function of the catalog. // The conceptual foundations of descriptive cataloging. Edited by Elaine Svenonius. San Diego [etc.] : Academic Press, 1989. p. 167-179.

Svenonius, E. (1992). Bibliographic entities and their uses. // Seminar on Bibliographic Records (1990. Stockholm). Seminar on Bibliographic Records: proceedings of the seminar held in Stockholm, 15-16 August 1990, and sponsored by the IFLA UBCIM Programme and the IFLA Division of Bibliographic Control. Edited by Ross Bourne. München [etc.] : Saur, 1992. p. 3-18.

Tillett, B. B. (1988). Bibliographic relationships in library catalogues. // International Cataloguing \& Bibliographic Control. 17 : 1 (1988) 3-6.

Tillett, B. B. (1989). Bibliographic structures: the evolution of catalog entries, references, and tracings. // The conceptual foundations of descriptive cataloging. Edited by Elaine Svenonius. San Diego [etc.] : Academic Press, 1989. p. 149-165.

Tillett, B. B. (1991a). A summary of the treatment of bibliographic relationships in cataloging rules. // Library Resources \& Technical Services. 35 : 4 (1991) 393-405.

Tillett, B. B. (1991b). A taxonomy of bibliographic relationships. // Library Resources \& Technical Services. 35 : 2 (1991) 150-158.

Tillett, B. B. (1992). Bibliographic relationships: an empirical study of the LC machine readable records. // Library Resources \& Technical Services. 36 : 2 (1992) 162-188.

UNIMARC: universal MARC format (1980). Recommended by the IFLA Working Group on Content Designators; set up by the IFLA Section on Cataloguing and the IFLA Section on Mechanization. 2nd ed. rev. London: IFLA International Office for UBC, 1980.

UNIMARC manual (1987). Edited by Brian P. Holt with the assistance of Sally H. McCallum \& A. B. Long. London : IFLA Universal Bibliographic Control and International MARC Programme, British Library Bibliographic Services, 1987.

Van Houten, S. (1981). In the iron age of cataloging. // Library Resources \& Technical Services. $25: 4$ (1981) 362-373.

Vellucci, S. L. (1990). Uniform titles as linking devices. // Cataloging \& Classification Quarterly. 12:1 (1990) 35-62. 
Weintraub, D. K. (1979). The essentials or desiderata of the bibliographic record as discovered by research. // Library Resources \& Technical Services. 23 : 4 (1979) 390402.

Scire. 2 : 2 (jul.-dic. 1996) 53-77 